\title{
IMPROVED ANGULAR DISCRETIZATION AND ERROR ANALYSIS OF THE LATTICE BOLTZMANN METHOD FOR SOLVING RADIATIVE HEAT TRANSFER IN A PARTICIPATING MEDIUM
}

\author{
A. F. Di Rienzo*, P. Asinari*1, R. Borchiellini*, S. C. Mishra** \\ *Department of Energetics, Politecnico di Torino, Turin - 10129, Italy \\ **Department of Mechanical Engineering, Indian Institute of Technology Guwahati, Guwahati -
} 781039, India

\begin{abstract}
PURPOSE - In this paper, some improvements to the lattice Boltzmann method (LBM) for solving radiative heat transfer in a participating medium are presented and validated. Validation of the model is performed by investigating the effects of spatial and angular discretizations and extinction coefficient on the solution. The error analysis and the order of convergence of the scheme are also reported.
\end{abstract}

DESIGN/METHODOLOGY/APPROACH - LB scheme is derived from the radiative transfer equation, where isotropic scattering and radiative equilibrium condition are assumed. Azimuthal angle is discretized according to the lattice velocities on the computational plane, while, concerning the polar angle, an additional component of the discrete velocity normal to the plane is introduced. Radiative LB scheme is used to solve a 2-D square enclosure benchmark problem. In order to validate the model, results of LB scheme are compared with a reference solution obtained through a Richardson extrapolation of the results of a standard Finite Volume Method (FVM).

FINDINGS - The proposed improvements drastically increase the accuracy of the previous method. Radiative LB scheme is found to be (at most) first order accurate. Numerical results show that solution gets more accurate when spatial and azimuthal angle discretizations are improved, but a saturation threshold exists. With regard to polar angle, minimum error occurs when a particular subdivision is considered.

ORIGINALITY/VALUE - This paper provides simple but effective improvements to the recently proposed lattice Boltzmann method for solving radiative heat transfer in a participating medium.

\footnotetext{
${ }^{1}$ Corresponding author: Tel: +39-011-564-4520, Fax: +39-011-564-4499, E-mail: pietro.asinari@ polito.it
} 


\section{NOMENCLATURE}

$\vec{e} \quad$ - velocity on the lattice

$\vec{E} \quad$ - total velocity

G - incident radiation

I - intensity

$N$ - number of subdivisions

$p$ - phase function

$q \quad$ - heat flux

$s$ - geometric distance

$T$ - temperature

$t$ - time

$X, Y$ - dimensions of the square enclosure

$x, y$ - plane spatial directions

$W \quad$ - weight

\section{Greek Symbols}

$\beta \quad$ - extinction coefficient

$\gamma$ - polar angle

$\delta$ - azimuthal angle

$\varepsilon \quad$ - energy shell constant

$\kappa_{a}-$ absorption coefficient

$\sigma \quad$ - Stefan-Boltzmann constant $=5.67 \times 10^{-8} \mathrm{~W} / \mathrm{m}^{2} \mathrm{~K}$

$\sigma_{s} \quad$ - scattering coefficient

$\tau$ - relaxation time

$\Psi \quad$ - dimensionless total heat flux

$\psi \quad$ - dimensionless heat flux

$\Omega \quad-$ angular direction

$\Delta \Omega$ - solid angle

$\Delta t \quad$ - time step

$\Delta \mathrm{x} \quad$ - space grid along $x$ axis 
$\Delta y \quad$ - space grid along $y$ axis

$\Delta 8$ - discrete azimuthal angle

$\Delta \gamma$ - discrete polar angle

\section{Subscripts}

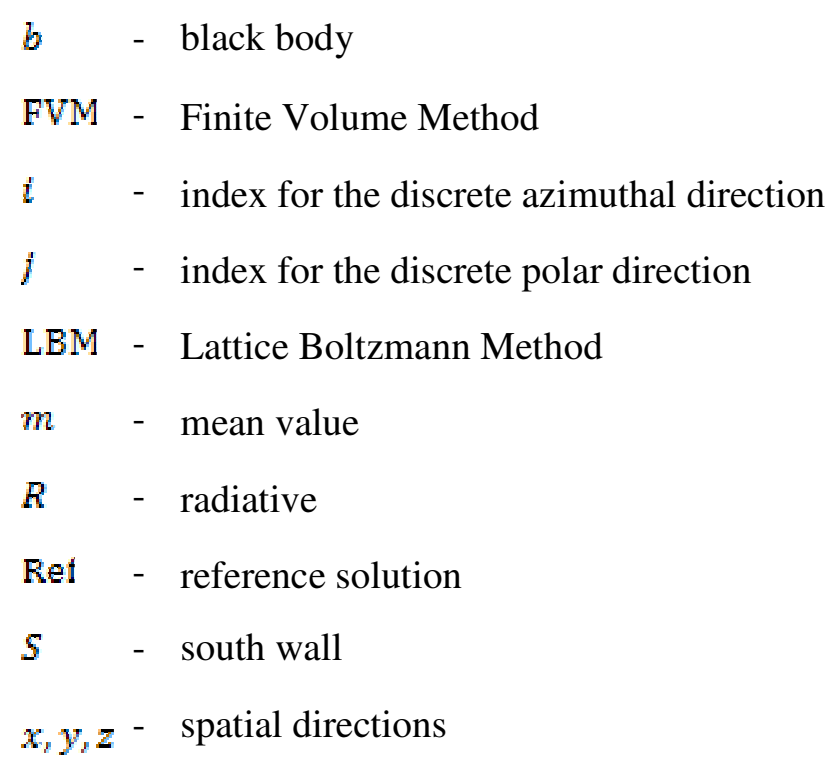

\section{Superscript}

$e q$ - equilibrium

\section{INTRODUCTION}

Consideration of volumetric radiation is important in many high temperature thermal devices and processes [1,2]. Design of boilers, furnaces, internal combustion engines and insulations are some of the systems which require a correct analysis of thermal radiation [1,2]. Analysis of phase change process of semitransparent materials such as glass and semiconductor materials requires knowledge of the volumetric radiation [3-6]. Correct estimates of volumetric radiation is also important in weather forecasting which relies on atmospheric radiation budget [7] and medium characterization of an optically participating medium like human tissue and laser surgery of a human organ [8-9]. Radiative transport through a participating medium is a volumetric phenomenon $[10,11]$. Unlike conduction and convection modes of heat transfer which depend on spatial and temporal dimensions, an analysis of radiation involves an additional three dimensions, viz., two angular dimensions (polar and azimuthal angles) and one spectral dimension. A mandatory consideration of two angular dimensions in all problems except the simplest case of the planar geometry in which case radiation is azimuthally symmetric and thus it depends only on the planar angle, the problems 
are difficult to analyze. In a conduction-convection and radiation problem, it is the computation of radiative component that is the most time consuming one. This excessive computational time in the computation of radiative information is for the reason that apart from covering all the spatial grid points in the solution domain, intensities at every grid point need to be traced from their points of origin in the enclosure to the grid point under consideration. At every grid point, intensities are spanned over the $4 \pi$ spherical space. A method becomes computationally more and more expensive if for a given number of control volumes, it requires more number of discrete directions.

The available numerical radiative transfer methods such as the flux method [10,11], the zonal method [10,11], the spherical harmonics method [10,11], the discrete ordinates method (DOM) [12,13], the discrete transfer method (DTM) [14-16], the collapsed dimension method [17] and the finite volume method (FVM) [18-20], in some form or the other, aim at minimizing the angular dependency of radiation in their formulations. Since the angular dependency cannot be fully eliminated, a method which is less prone to ray effect and is compatible to other CFD solvers such as the finite difference method (FDM) and the FVM for solving the combined mode problems in simple to complex geometry are the most desirable ones. Among the existing numerical radiative transfer methods, the FVM [18-20] is the most robust one. This is not only for the reason that the development of the FVM is the latest in the series, but for the very reason that it adopts the same principles of the FVM that has been widely used in the analysis of fluid flow and heat transfer problems. Further, unlike the DOM $[12,13]$, the FVM is fully conservative. In this, the ray effect is the minimal. However, even with the FVM, radiation still remains a computationally expensive component. Therefore, search for a computationally more efficient method still continues.

The lattice Boltzmann method (LBM) [21, 22] is relatively a recent computational tool which has found widespread applications in science and engineering. This method is viewed as a potential versatile CFD tool. Since in the LBM, processes are localized, it is well suited for a parallel architecture. In the recent past, the LBM has been applied to a large class of fluid flow and heat transfer problems [22]. Application of the LBM to solve energy equations, in particular by means of the so-called passive scalar approach [23-28], has been known for quite some time. This has essentially been the simplest approach in which the temperature is treated as a passive scalar, which is diffused and moderately advected by the flow velocity. This particular approach has been adopted to analyze several thermal problems [23-28] that involved computations of the density, velocity and temperature fields caused by convection and/or conduction heat transfer. Those studies, did not consider the effect of volumetric radiation which is an important component in high temperature applications. 
Mishra and co-workers [6, 20, 29-32] have applied the LBM to solve heat transfer problems involving thermal radiation. However, in such problems, the volumetric thermal radiation was always computed using the conventional numerical radiative transfer methods such as the DOM $[12,13]$, the DTM [14-16], the collapsed dimension method [17] and the FVM [18-20]. The previous studies [6, 20,29-32] have shown the superiority of the LBM over the FDM and the FVM to solve the energy equations of heat transfer problems involving thermal radiation. However, in none of the previous studies, the computation of radiative information, which is the main time consuming component, has been computed using the LBM, and thus, the usage of the LBM for the analysis of radiative transport problems has not been investigated before. Further, in the combined mode problems studied in references [6, 20, 29-32], the computational grids of the conventional radiation solvers such as the DTM [14-16], the DOM [12, 13], the FVM [18-20], etc., have always been different from the lattices of the LBM. Thus, the radiative information computed using these methods required to be interpolated to the lattice nodes that required an additional computational step.

Very recently a different approach has been proposed by Asinari et al. [33], where the LBM is directly used to solve the radiative heat transfer in a participating medium. Essentially the idea is to interpret the (transient) radiative transfer equation (RTE) as a sort of kinetic equation and consequently to solve it directly by means of the standard LBM formalism. The numerical implementation is very simple and it is particularly suitable for complex geometries. The reason is twofold: the intensities at every grid point are automatically traced from their points of origin at the solid walls and the data structures for radiation can be the same of those for fluid flow. These advantages make promising the application of this approach to porous media. However the accuracy of the method still needs to be investigated and further improved for making it competitive with standard FVM radiation solvers. This paper aims to suggest simple but effective improvements to the method proposed in Ref. [33].

The paper is organized as follows. The next section about the formulation reports the essential steps of the proposed method, including the further improvements concerning the angular discretization of the radiative transfer equation. Moreover a detailed error analysis is reported aiming to point out the dependence of the numerical error on the discretization parameters. The following section reports the numerical results for the 2-D rectangular enclosure (both in terms of temperature field and radiative heat flux). In particular the complete simulation plan, the convergence study and the attempts to reduce the computational demand (the so-called thermalization of intensities out of the main simulation plane) are presented. The last section reports the concluding remarks. Finally, in 
appendix, the numerical results obtained by standard FVM and used for comparison are reported for sake of completeness.

\section{FORMULATION}

Let us consider the 2-D square enclosure given in Fig. 1. The participating medium bounded by the enclosure is assumed to be homogeneous, absorbing, emitting and scattering. All the boundaries are diffusive and gray: the source of radiation is the south wall at temperature $T_{s}$, while the other three boundaries are cold.

The starting point for deriving the LBM formulation is the Radiative Transfer Equation (RTE). In any direction $\vec{s}$ RTE reads:

$$
\frac{d I}{d s}=\vec{s} \cdot \nabla I=-\beta I+\kappa_{a} l_{b}+\frac{\sigma_{s}}{4 \pi} \int_{h \pi} I p\left(\Omega, \Omega^{\prime}\right) d \Omega^{\prime},
$$

where $I$ is the intensity, $\kappa_{a}$ is the absorption coefficient, $I_{b}-o T^{4} / u$ is the blackbody intensity, $\beta$ is the extinction coefficient, $\sigma_{s}$ is the scattering coefficient and $p$ is the scattering phase function. $s$ is the distance in the direction $\vec{s}$, which is defined as:

$$
\vec{s}=(\sin \gamma \cos 0) \vec{l}+(\sin \gamma \sin 0) \vec{j}+\cos \gamma \vec{k}
$$

where $\gamma$ is the polar angle and $\delta$ is the azimuthal angle.

Since radiation is the only heat transfer mechanism under investigation, radiative equilibrium condition can be considered, so that $\nabla \cdot \vec{q}_{k}=0$, where $\vec{q}_{k}$ is the radiative heat flux and its divergence is defined as follows:

$$
\nabla \cdot \vec{q}_{k}=\kappa_{a}\left(4 \pi I_{b}-G\right)
$$

Thus, radiative equilibrium condition implies that the volumetric absorption $f$ equals the volumetric emission $4 \pi I_{b}$. If scattering is assumed isotropic $(p=1)$, Eq. (1) can be rewritten as:

$$
\frac{d I}{d s}=\vec{s} \cdot \nabla I=-\beta I+\left(\kappa_{a}+\sigma_{s}\right) \frac{G}{4 \pi}=\beta\left(\frac{G}{4 \pi}-I\right)
$$

since the extinction coefficient $\beta=F_{a}+\sigma_{S}$. For discrete directions, Eq. (2) is written as:

$$
\frac{d I_{i j}}{d s}=\vec{s}_{i j} \cdot \nabla I_{i j}=\beta\left(\frac{G}{4 \pi}-I_{i j}\right)
$$

where $I_{i j}$ is the intensity evaluated along the $i$-th azimuthal direction and the $j$-th polar direction. 


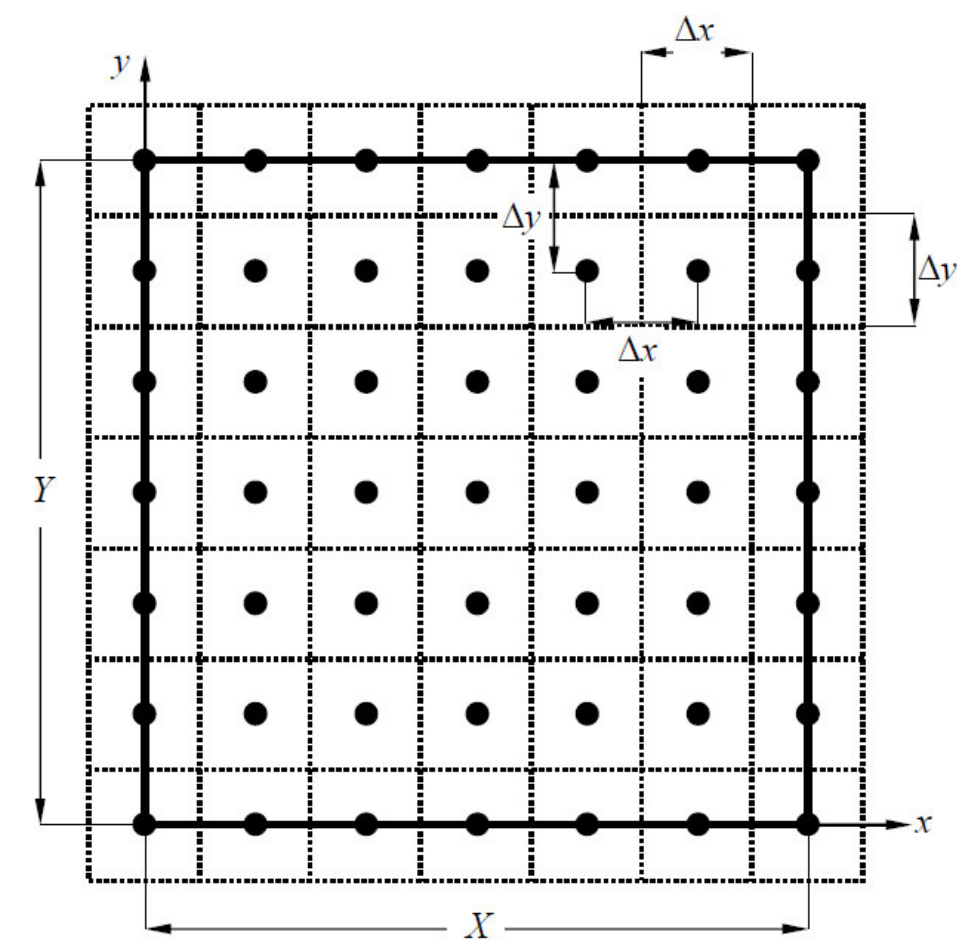

Figure 1. 2-D square enclosure and computational grid

In the LBM formulation proposed by Asinari et al. [33], isotropy in the polar direction is assumed and angular dependence of the intensity is only due to the azimuthal direction. Azimuthal angle is discretized (Fig. 2) by introducing a finite number of discrete velocities $\vec{e}_{\lambda, i}\left(\lambda=x_{\imath} y\right)$, lying on the lattice, whose magnitude is given by $e_{i}=\sqrt{e_{x, i}^{2}+e_{y, i}^{2}}$. For a D2Q32 lattice (Fig. 2), velocities are defined as:

$$
\begin{aligned}
& e_{1,3}=( \pm 1,0) U \quad e_{2,4}=(0, \pm 1) U \quad e_{5,7,2}=( \pm 1, \pm 1) U \\
& e_{9,12}=( \pm 2,1) U \quad e_{13,16}=(\mp 2,-1) U \\
& e_{10,11}=( \pm 1,2) U \quad e_{14,15}=\left(\mp_{1},-2\right) U \\
& e_{17,24}=( \pm 3,1) U \quad e_{25,32}=\left(\mp_{3,-1}\right) U \\
& e_{18,23}=( \pm 3,2) U \quad e_{26,31}=(\mp 3,-2) U \\
& e_{19,22}=( \pm 2,3) U \quad e_{27,30}=(\mp 2,-3) U \\
& e_{20,21}=( \pm 1,2) U \quad e_{28,29}=( \pm 1,-3) U
\end{aligned}
$$

where $U=\Delta x / \Delta t$ is the speed and it has been assumed $\Delta x=\Delta y$. It is to be noted that the directions 1-8 correspond to the D2Q8 lattices, 1-16 correspond to the D2Q16 lattice and for the D2Q32 lattices, the directions are 1-32. It is to be further noted that in the D2Q32 lattice, 5 different energy shells exist, and for directions 1, 5, 9, 17 and 18, the magnitudes of the propagation velocities are $U, U \sqrt{2}, U \sqrt{5}, U \sqrt{10}$ and $U \sqrt{13}$, respectively. In general, this means that we can express the magnitude of the lattice velocity as $\varepsilon_{i}=\varepsilon_{i} U$, where $\varepsilon_{i}$ is a constant depending on the energy shell of the considered velocity. 
Even though not discretizing the polar angle saves a lot of computations, this approximation may spoil the accuracy of the method, particularly in case of refined computational grids, where a sort of saturation of the numerical error appears [33]. Hence, in the present paper, a different solution is proposed and the polar angle $\gamma(0 \leq \gamma \leq \pi)$ is discretized as well. In this paper discretization of the polar angle has been performed by introducing along the z-axis a velocity component $e_{z, j}$ of the discrete lattice velocity, which has been designed in such a way that the projection on the lattice of the total velocity $\vec{E}=\left(e_{x, i}, e_{y, i}, e_{x_{i j}}\right)$ overlaps the velocity on the lattice $\vec{e}_{\lambda, i}$. Hence, $e_{\sigma_{i, j}}$ is defined as follows:

$$
e_{a_{i j}}=\tan \left(\frac{\pi}{2}-\gamma_{j}\right) e_{i s}
$$

where $\gamma_{j}$ is the discrete polar angle in the $j$-th direction, so that the magnitude of the total velocity is:

$$
E_{i j}=\sqrt{e_{x, i}^{2}+e_{y, i}^{2}+e_{z, j}^{2}}
$$

The regions of influence of the PDFs in the solution plane for D2Q8 and D2Q16 lattices are shown in Figs. 3a and 3b. In the D2Q8 lattice, the azimuthal angle is discretized into 8 divisions and all directions are equally spaced (Fig. 3a). In D2Q16, 8 more directions are introduced keeping the D2Q8 lattice directions fixed. Similarly, the D2Q32 lattice is obtained by adding 16 more directions to the ones of the D2Q16 lattice. While in the D2Q8 lattice the azimuthal angle is uniformly discretized, in the D2Q16 (Fig. 3b) and D2Q32 lattices the regions of influence of all the PDFs are not the same and the azimuthal angle is not discretized uniformly.

In the polar direction, due to the definition of the z-axis velocity component $e_{s_{i, j}}$, regions of influence of the PDFs are all equally spaced, as shown in Figs. $4 \mathrm{a}$ and $4 \mathrm{~b}$, where 4 and 8 subdivisions are reported. 


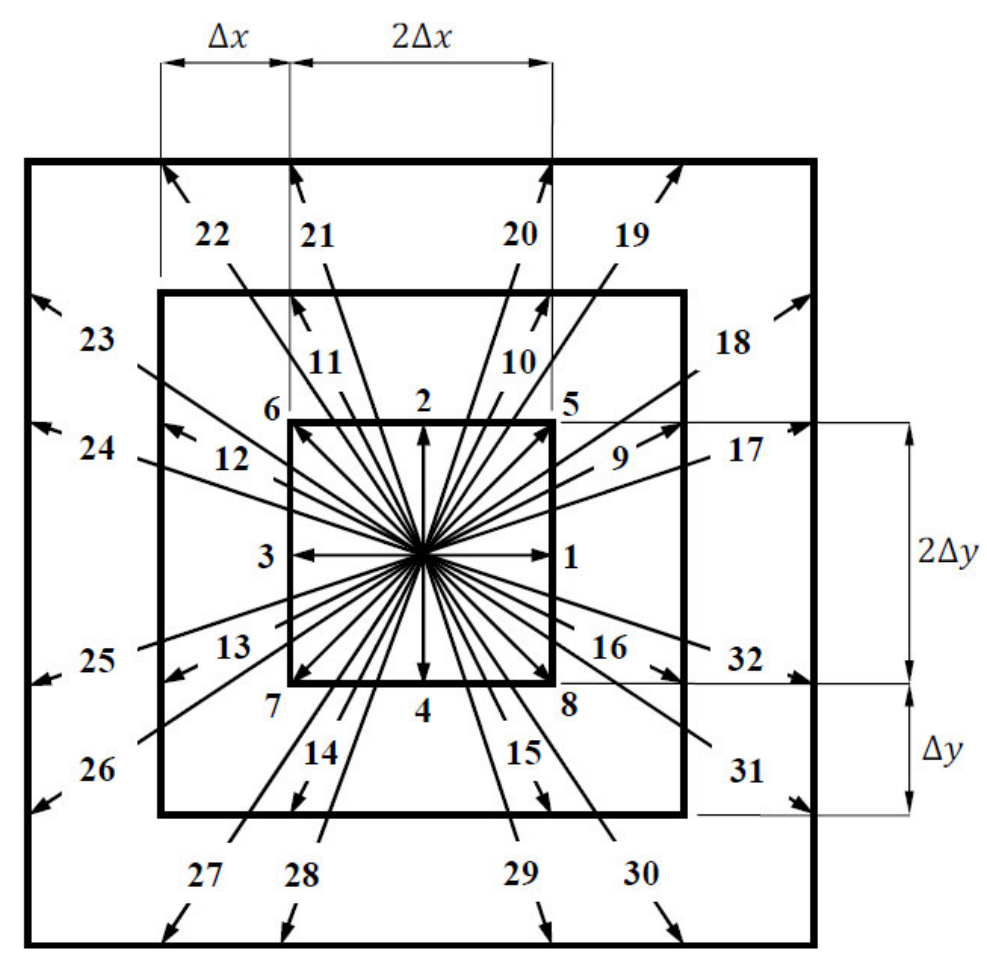

Figure 2. Schematic of the lattice: azimuthal angle discretization

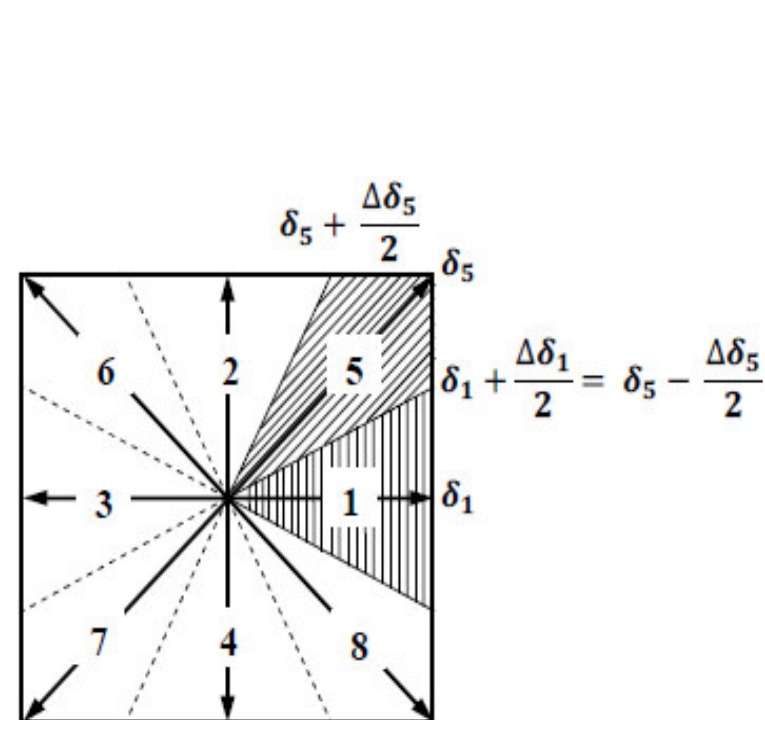

(a)

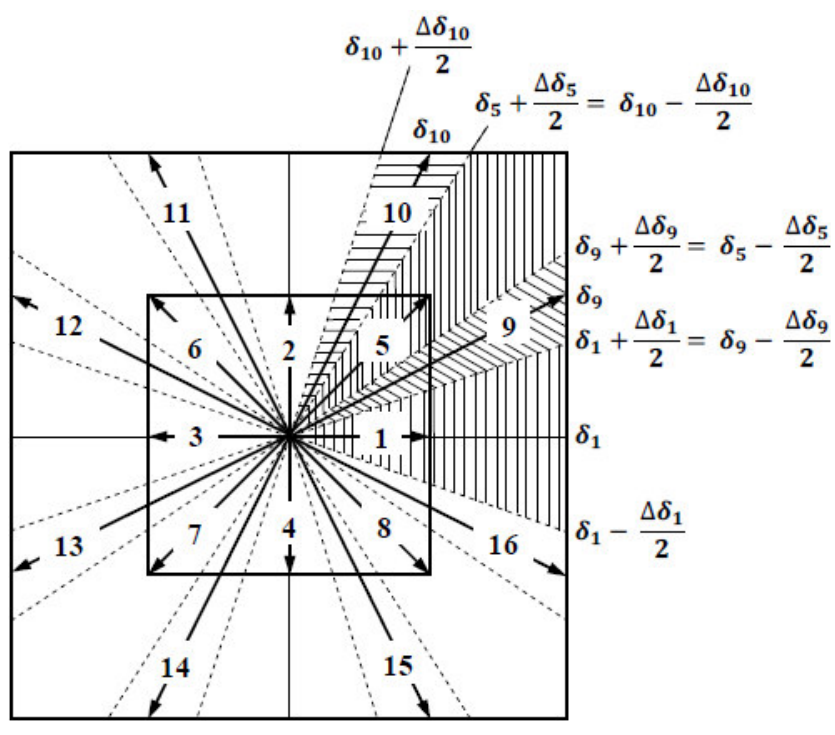

(b)

Figure 3. Regions of influence of the particle distribution function for D2Q8 and D2Q16 lattices. 


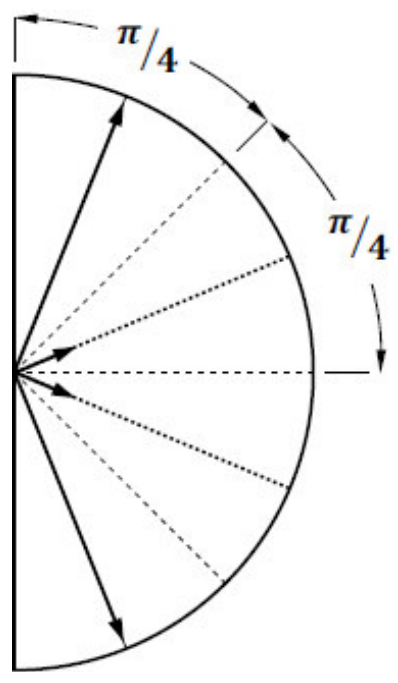

(a)

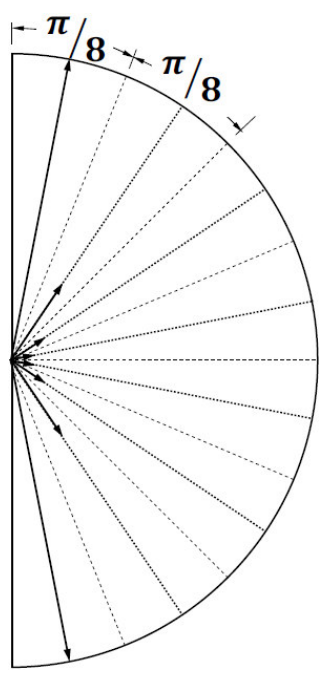

(b)

Figure 4. Regions of influence of the particle distribution function for D2Q8 and D2Q16 lattices.

Time dependence of the intensity is usually neglected in radiation problems because the speed of light is much faster than heat transfer phenomena. However in this case, it is convenient to consider a pseudo-transient equation as the starting point of the LBM formalism. The transient RTE reads:

$$
\frac{1}{c} \frac{\partial I_{i j}}{\partial t}+\vec{s}_{i j} \cdot \nabla I_{i j}=\beta\left(\frac{G}{4 \pi}-I_{i j}\right)
$$

where $c$ is the speed of light. Actually, the starting point for the derivation of the radiative LB scheme is Eq. (6), where we assume, along each discrete direction, the fictitious speed of light to be equal to the corresponding microscopic velocity $c=E_{i j}^{v}$. Hence, from the LBM point of view, Eq. (6) rewrites as:

$$
\frac{1}{E_{i j}} \frac{\partial I_{i j}}{\partial t}+\vec{s}_{i j} \cdot \nabla I_{i j}=\beta\left(\frac{G}{4 \pi}-I_{i j}\right) .
$$

Multiplying Eq. (7) by $E_{i j}$ yields:

$$
\frac{\partial I_{i j}}{\partial t}+\vec{E} \cdot \nabla I_{i j}=\frac{\partial I_{i j}}{\partial t}+e_{w, i} \frac{\partial I_{i j}}{\partial x}+e_{y, i} \frac{\partial I_{i j}}{\partial y}+e_{z i j} \frac{\partial I_{i j}}{\partial z}=E_{i j} \beta\left(\frac{G}{4 \pi}-I_{i j}\right)
$$

Recalling that $\partial I_{i} / \partial z=0$ by definition of 2-D problem, one can rewrite the discrete Boltzmann equation for a 2-D radiative problem:

$$
\frac{\partial I_{i j}}{\partial t}+\vec{e}_{i} \cdot \nabla I_{i j}-\frac{D I_{i j}}{D t}-E_{i j} \rho\left(\frac{G}{4 \pi}-I_{i j}\right),
$$

where $i=1_{, \ldots,} N_{\delta}$ and $j=1_{2} \ldots, N_{Y} . N_{\delta}$ and $N_{\gamma}$ are the total number of discrete subdivisions for the azimuthal and polar angles, respectively. 
In order to obtain the usual LBM formulation, applying the forward Euler approximation to Eq. (9) yields:

$\frac{I_{i j}\left(\vec{r}_{n}^{*}+\vec{e}_{i} \Delta t, t+\Delta t\right)-I_{i j}\left(\vec{r}_{n}, t\right)}{\Delta t}-\frac{1}{\tau_{i j}}\left[I_{i j}^{\theta q}\left(\vec{r}_{n}^{*}, l\right)-I_{i j}\left(\vec{T}_{n}, l\right)\right]+O(\Delta l)+O\left(e_{i} \Delta l\right)$

where the relaxation time $\tau_{i j}=1 / \beta E_{i j}$. According to LBM terminology, $I_{i j}$ is the particle distribution function (PDF) and it is the carrier of the radiative energy. $I_{i j}^{* a}$ is the equilibrium distribution function, defined as:

$$
I_{i j}^{* q}=\frac{G}{4 \pi}=\frac{1}{4 \pi} \int_{\Omega=0}^{4 \pi} I\left(\vec{r}_{n}, t, \delta, \gamma\right) d \Omega=\frac{1}{4 \pi} \int_{0}^{2 \pi} \int_{0}^{\pi} I\left(\vec{r}_{n}, t, \delta, \gamma\right) \sin \gamma d \delta d \gamma
$$

Integral in Eq. (11) is solved by means of quadrature schemes. Let us consider a portion of sphere of the size of $\left[\delta_{i}-\frac{\Delta \delta_{i}}{2}, \delta_{i}+\frac{\Delta \delta_{i}}{2}\right]$ and $\left[\gamma_{j}-\frac{\Delta \psi_{Y}}{2}, \gamma_{j}+\frac{\Delta Y}{2}\right]$ with respect to the azimuthal and polar directions, respectively. Taylor expanding the intensity around the value at the centroid of the infinitesimal region under consideration yields:

$$
I\left(\vec{r}_{n}, t_{,}, \gamma\right)=I\left(\vec{r}_{n}, t_{i} \delta_{i} \gamma_{j}\right)+o\left(\max _{\mathrm{i}}\left(\Delta \hat{\delta}_{i}\right)\right)+o(\Delta \gamma)
$$

and the integral over the same region is:

$$
\begin{aligned}
& \delta_{i}+\frac{\Delta \delta_{i}}{2} \gamma_{j}+\frac{\Delta \gamma}{2} \quad \delta_{i}+\frac{\Delta \delta_{j}}{2} \gamma_{j}+\frac{\Delta \gamma}{2} \\
& \int_{\delta_{1}-\frac{\Delta \delta_{i}}{2}} \int_{\gamma_{j}-\frac{\Delta \gamma}{2}} I\left(\vec{r}_{n}, t, \delta_{,} \gamma\right) \sin \gamma d \delta d \gamma=\int_{\delta_{1}-\frac{\Delta \delta_{i}}{2}} \int_{\gamma_{j}-\frac{\Delta \gamma}{2}} I\left(\vec{r}_{n}, t, \delta_{i}, \gamma_{j}\right) \sin \gamma d \delta d \gamma+o\left(\max _{i}\left(\Delta \delta_{i}^{2}\right) \Delta \gamma\right) \\
& +o\left(\max _{i}\left(\Delta \delta_{i}\right) \Delta \gamma^{2}\right) \\
& \delta_{i}+\frac{\Delta \delta_{i}}{2} y_{j}+\frac{\Delta y}{2} \\
& =I_{i j} \int_{\delta_{i}-\frac{\Delta \delta_{i}}{2}} \int_{\gamma_{j}-\frac{\Delta \gamma}{2}} \sin \gamma d \delta d \gamma+o\left(\max _{i}\left(\Delta \delta_{i}^{2}\right) \Delta \gamma\right)+o\left(\max _{i}\left(\Delta \varepsilon_{i}\right) \Delta \gamma^{2}\right)
\end{aligned}
$$

If Eq. (13) is extended to the whole solid angle, the equilibrium PDF can be computed as follows:

$$
\begin{aligned}
& I_{i j}^{ \pm q}=\frac{1}{4 \pi} \int_{0}^{2 \pi} \int_{0}^{\pi} I\left(\vec{r}_{n}, t, \delta, \gamma\right) \sin \gamma d \delta d \gamma \\
& =\sum_{i=1}^{N_{i}} \sum_{j=1}^{N_{\gamma}}\left[I_{i j} \int_{\delta_{i}-\frac{\delta_{i}}{2}}^{\delta_{i}+\frac{\Delta \delta_{i}}{2}} \int_{\gamma_{j}-\frac{\Delta \psi}{2}} \frac{\Delta \gamma}{4 \pi} \sin \gamma d \delta d \gamma\right]+o\left(\max _{i}\left(\Delta \delta_{i}\right)\right)+o(\Delta \gamma) \\
& =\sum_{i=1}^{N_{\theta}} \sum_{j=1}^{N_{\gamma}} w_{i j} I_{i j}+o\left(\max _{i}\left(\Delta \delta_{i} j\right)\right)+o(\Delta \gamma) \text {. }
\end{aligned}
$$


where $W_{i j}$ is the weight corresponding to the discrete directions $i$ and $j$ and is defined as:

$$
W_{i j}=\frac{1}{4 \pi} \int_{\gamma_{j}-\frac{\Delta \gamma}{2}}^{\gamma_{j}+\frac{\Delta \gamma}{2}} \int_{\delta_{\mathrm{i}}-\frac{\Delta \delta_{\mathrm{i}}}{2}}^{\delta_{\mathrm{i}}+\frac{\Delta \delta_{i}}{2}} \sin \gamma d \delta d \gamma
$$

The algorithm to solve Eq. (7) is usually split into two parts, which are called collision and streaming and are given by the following equations, respectively:

$$
\begin{gathered}
I_{i j}^{*}\left(\vec{r}_{n}, t\right)=I_{i j}\left(\vec{r}_{w}, t\right)+\frac{\Delta t}{\tau_{i j}}\left[I_{i j}^{\theta q}\left(\vec{r}_{n}, t\right)-I_{i j}\left(\vec{r}_{n}, t\right)\right], \\
I_{i j}\left(\vec{r}_{n}+\vec{e}_{i} \Delta t, t+\Delta t\right)=I_{i j}^{*}\left(\vec{r}_{n}, t\right) .
\end{gathered}
$$

Collision step assign values to each node from the values of the nodes in the local neighborhood; streaming step propagates the state of each node to the neighboring nodes along directions given by the lattice velocities $\vec{e}_{i}$. Eqs. (16) and (17) provides the numerical solution for Eq. (9). The global numerical error can be determined by collecting the errors due to forward Euler and to the quadrature schemes given by Eq. (10) and Eq. (12) respectively, namely

$$
\begin{aligned}
& \frac{I_{i j}\left(\vec{r}_{n}+\vec{e}_{i} \Delta t, t+\Delta t\right)-I_{i j}\left(\vec{r}_{n}, t\right)}{\Delta t} \\
& \quad=\frac{1}{\tau_{i j}}\left[I_{i j}^{\theta q}\left(\vec{r}_{n}, t\right)-I_{i j}\left(\vec{r}_{n}, t\right)\right]+o(\Delta t)+o\left(e_{i} \Delta t\right)+o\left(\max _{i}\left(\Delta \delta_{i}\right)\right)+o(\Delta \gamma) .
\end{aligned}
$$

Recalling that $e_{i}=\varepsilon_{i} U$, where $\varepsilon_{i}$ is a constant depending on the energy shell of the considered velocity, assuming $U-\Delta x / \Delta t$ as a constant (depending on the stability region), the global error becomes:

$$
E r r=o\left(\left(1+\varepsilon_{i}\right) \Delta x\right)+o\left(\max _{i}\left(\Delta \hat{\delta}_{i}\right)\right)+o(\Delta \gamma)
$$

It is evident from the previous expression that the dependence of the global error on the discretization parameters is not trivial. In fact, improving the discretization of the azimuthal angle, i.e. reducing $\max _{i}\left(\Delta \delta_{i}\right)$, forces one to consider larger lattices, with larger energy shells, which usually spoil the accuracy of the advection step (because of larger $\varepsilon_{i}$ ). On the other hand, accurate advection step requires a compact computational stencil, i.e. few energy shells, but this makes quite rough the discretization of the azimuthal angle and consequently the computation of the collision step (by the definition of local equilibrium). With other words, because of the geometrical 
construction, the following relation holds $\varepsilon_{i} \propto 1 / \max _{i}\left(\Delta \delta_{i}\right)$. Hence there is a tradeoff between the accuracy of the advection step and that of the collision step, which both affect the global error.

\section{NUMERICAL RESULTS}

The proposed LB scheme has been applied to solve a 2-D square enclosure problem. In this work it has been assumed $X=Y=1$ and uniform spatial discretization along both $x$ and $y$ directions. In order to validate the LBM formulation, FVM results have been considered benchmark. Since both FVM and LBM are iterative methods, a convergence criterion is required: FVM and LBM solutions are assumed to converge when the maximum change in the incident radiation at any point is less than $1 \times 10^{-10}$ between two successive iterations. Validation of the LBM formulation has been performed for several values of the spatial and angular discretizations and extinction coefficients, as shown in Table 1. Spatial discretization has been chosen in such a way that the Knudsen number is smaller than a threshold value that ensures stability of the LBM solution. In this work it has been chosen

$$
K n=\beta \Delta x \leq 0.05
$$

where $\Delta x=X / N_{x}$ is the spatial grid length and $N_{x}$ represents the number of nodes along $x$ axis.

Table 1. Summary of the parameters adopted in the validation analysis..

\begin{tabular}{|c|c|}
\hline $\boldsymbol{\beta}=\mathbf{2 . 0}$ & $\boldsymbol{\beta}=\mathbf{5 . 0}$ \\
\hline $\mathrm{N}_{\mathrm{x}}=\mathrm{N}_{\mathrm{y}}=[40,80,160]$ & $\mathrm{N}_{\mathrm{x}}=\mathrm{N}_{\mathrm{y}}=[100,200,400]$ \\
$\mathrm{N}_{\delta}=[8,16,32]$ & $\mathrm{N}_{\delta}=[8,16,32]$ \\
$\mathrm{N}_{\gamma}=[1,4,8,16]$ & $\mathrm{N}_{\gamma}=[1,4,8,16]$ \\
\hline
\end{tabular}

Table 2. Summary of the parameters adopted in the convergence analysis.

\begin{tabular}{|c|c|}
\hline $\boldsymbol{\beta}=\mathbf{2 . 0}$ & $\boldsymbol{\beta}=\mathbf{5 . 0}$ \\
\hline $\mathrm{N}_{\mathrm{x}}=40, \mathrm{~N}_{\delta}=8, \mathrm{~N}_{\gamma}=4$ & $\mathrm{~N}_{\mathrm{x}}=100, \mathrm{~N}_{\delta}=8, \mathrm{~N}_{\gamma}=4$ \\
$\mathrm{~N}_{\mathrm{x}}=80, \mathrm{~N}_{\delta}=16, \mathrm{~N}_{\gamma}=8$ & $\mathrm{~N}_{\mathrm{x}}=200, \mathrm{~N}_{\delta}=16, \mathrm{~N}_{\gamma}=8$ \\
$\mathrm{~N}_{\mathrm{x}}=160, \mathrm{~N}_{\delta}=32, \mathrm{~N}_{\gamma}=16$ & $\mathrm{~N}_{\mathrm{x}}=400, \mathrm{~N}_{\delta}=32, \mathrm{~N}_{\gamma}=16$ \\
\hline
\end{tabular}

The temperature of the participating medium is given by the following equation:

$$
T=\left(\frac{G}{4 \sigma}\right)^{1 / 4},
$$


where the volumetric absorption $G$ is computed from Eq. (11). Fig. 5 shows the distribution of the temperature in the 2-D domain. Tables 3 shows the mean temperature $T_{m}$ of the participating medium inside the enclosure:

$$
T_{m}=\frac{1}{X Y} \int_{0}^{X} \int_{0}^{Y} T(x, y) d x d y
$$

which is related to the internal energy

$$
u^{*}=\frac{u}{c_{v}}=\frac{1}{X Y} \int_{0}^{x} \int_{0}^{y} T(x, y) d x d y=T_{m}
$$

if the specific heat at constant volume $c_{v}$ of the medium assumes a constant value. In Appendix the mean temperature $T_{m}$ evaluated by means of a standard FVM code is reported in Table A.1.

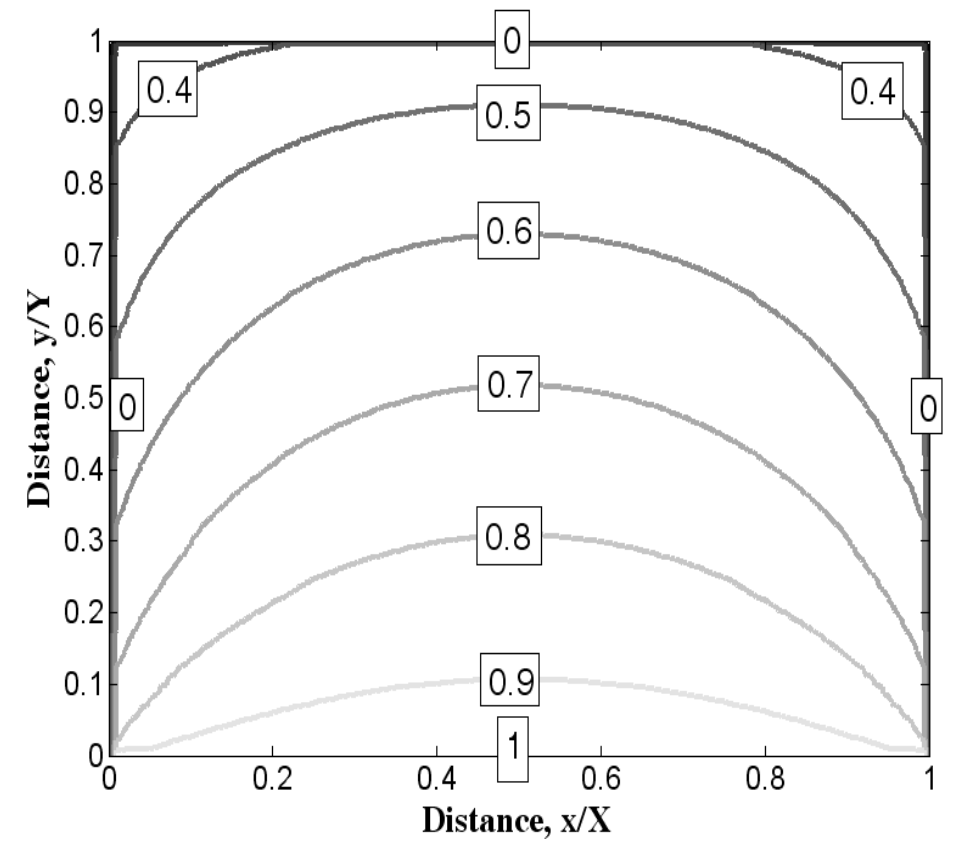

Figure 5. Distribution of the temperature in the square enclosure.

Table 3. Mean temperature of the medium inside the enclosure.

\begin{tabular}{|c|c|c|c|c|c|c|c|}
\cline { 3 - 7 } \multicolumn{2}{c|}{} & \multicolumn{3}{|c|}{$\boldsymbol{\beta}=\mathbf{2 . 0}$} & \multicolumn{3}{c|}{$\boldsymbol{\beta}=\mathbf{5 . 0}$} \\
\cline { 3 - 8 } \multicolumn{2}{c|}{} & $\mathbf{N}_{\mathbf{x}}=\mathbf{4 0}$ & $\mathbf{N}_{\mathbf{x}}=\mathbf{8 0}$ & $\mathbf{N}_{\mathbf{x}}=\mathbf{1 6 0}$ & $\mathbf{N}_{\mathbf{x}}=\mathbf{1 0 0}$ & $\mathbf{N}_{\mathbf{x}}=\mathbf{2 0 0}$ & $\mathbf{N}_{\mathbf{x}}=\mathbf{4 0 0}$ \\
\hline \multirow{3}{*}{$\mathbf{N}_{\delta}=\mathbf{8}$} & $\mathbf{N}_{\gamma}=\mathbf{1}$ & 0.63122 & 0.65742 & 0.67072 & 0.64538 & 0.65593 & 0.66123 \\
& $\mathbf{N}_{\gamma}=\mathbf{4}$ & 0.62774 & 0.65391 & 0.66718 & 0.64166 & 0.65223 & 0.65754 \\
& $\mathbf{N}_{\gamma}=\mathbf{8}$ & 0.62824 & 0.65442 & 0.66770 & 0.64204 & 0.65261 & 0.65793 \\
& $\mathbf{N}_{\gamma}=\mathbf{1 6}$ & 0.62832 & 0.65451 & 0.66779 & 0.64219 & 0.65276 & 0.65808 \\
\hline $\mathbf{N}_{\delta}=\mathbf{1 6}$ & $\mathbf{N}_{\gamma}=\mathbf{1}$ & 0.62911 & 0.65528 & 0.66855 & 0.64364 & 0.65427 & 0.65962 \\
& $\mathbf{N}_{\gamma}=\mathbf{4}$ & 0.62542 & 0.65157 & 0.66484 & 0.63989 & 0.65054 & 0.65590 \\
& $\mathbf{N}_{\gamma}=\mathbf{8}$ & 0.62589 & 0.65204 & 0.66531 & 0.64017 & 0.65083 & 0.65619
\end{tabular}




\begin{tabular}{|l|c|c|c|c|c|c|c|}
\hline & $\mathbf{N}_{\gamma}=\mathbf{1 6}$ & 0.62595 & 0.65211 & 0.66537 & 0.64025 & 0.65092 & 0.65628 \\
\hline \multirow{4}{*}{$\mathbf{N}_{\boldsymbol{\delta}} \mathbf{3 2}$} & $\mathbf{N}_{\gamma}=\mathbf{1}$ & 0.62785 & 0.65440 & 0.66787 & 0.64298 & 0.65387 & 0.65935 \\
& $\mathbf{N}_{\gamma}=\mathbf{4}$ & 0.62404 & 0.65062 & 0.66410 & 0.63911 & 0.65006 & 0.65557 \\
& $\mathbf{N}_{\gamma}=\mathbf{8}$ & 0.62451 & 0.65107 & 0.66456 & 0.63934 & 0.65030 & 0.65581 \\
& $\mathbf{N}_{\gamma}=\mathbf{1 6}$ & 0.62455 & 0.65112 & 0.66461 & 0.63939 & 0.65035 & 0.65586 \\
\hline
\end{tabular}

Overall radiative heat flux is computed from the following relation:

$$
q_{k}=\int_{0}^{2 \pi} \int_{0}^{\pi} I(\vec{n} \cdot \vec{s}) \sin \gamma d \delta d \gamma \text {. }
$$

In the present work, we are interested in the heat flux normal to the south (hot) wall and thus, we define:

$$
q_{F y}=\int_{0}^{2 \pi} \int_{0}^{\pi} i\left(\vec{r}_{n}, t, \delta, \gamma\right) \sin \delta \sin ^{2} \gamma d \delta d \gamma
$$

as the heat flux along $y$ faces of the enclosure. Applying the quadrature scheme already used in Eq. (14), Eq. (22) becomes:

$$
q_{R y}=\sum_{i=1}^{N_{N}} \sum_{j=1}^{N_{y}} W_{i j}^{y} I_{i j}
$$

where the weights are given by:

$$
w_{i j}^{y}=\int_{y_{j}-\frac{\Delta \gamma}{2}}^{\gamma_{j}+\frac{\Delta y}{2}} \sin ^{2} \gamma d \gamma \int_{\delta_{\mathrm{i}}-\frac{\Delta \delta_{\mathrm{i}}}{2}}^{\delta_{\mathrm{i}}+\frac{\Delta \delta}{2}} \sin \delta d \delta .
$$

Fig. 6 shows the distribution of the dimensionless heat flux along the $y$ direction:

$$
\psi_{y}=\frac{q_{N y}}{\sigma T_{s}^{4}}
$$

In Tables 4 dimensionless total heat flux along the south wall is reported as function of the parameters of Table 1. Dimensionless total heat flux evaluated by means of FVM code is reported in Table A.2. The dimensionless total heat flux at a given coordinate along the $y$ axis is:

$$
\Psi_{l y=y^{*}}=\int_{0}^{X} \psi_{y}(x, y) d x .
$$




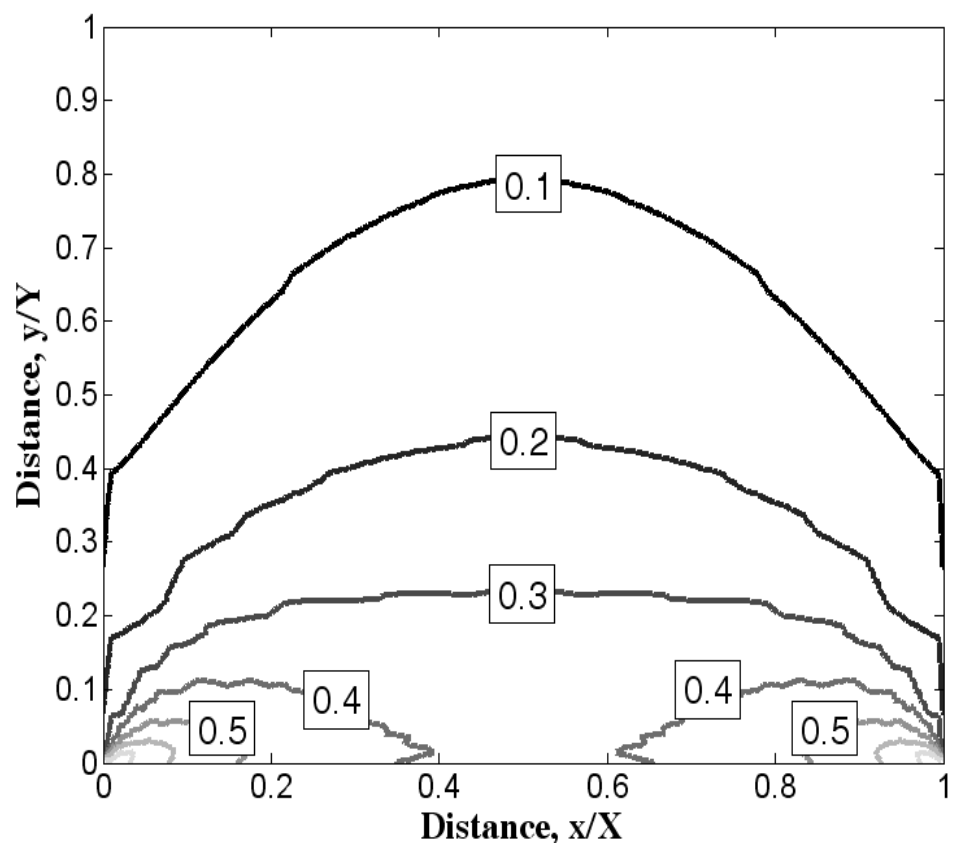

Figure 6. Distribution of dimensionless heat flux $\psi_{y}$ in the square enclosure.

Table 4. Dimensionless total heat flux along the south wall.

\begin{tabular}{|c|c|c|c|c|c|c|c|}
\cline { 3 - 8 } \multicolumn{2}{c|}{} & \multicolumn{3}{c|}{$\boldsymbol{\beta}=\mathbf{2 . 0}$} & \multicolumn{3}{c|}{$\boldsymbol{\beta}=\mathbf{5 . 0}$} \\
\cline { 3 - 8 } \multicolumn{2}{c|}{} & $\mathbf{N}_{\mathbf{x}}=\mathbf{4 0}$ & $\mathbf{N}_{\mathbf{x}}=\mathbf{8 0}$ & $\mathbf{N}_{\mathbf{x}}=\mathbf{1 6 0}$ & $\mathbf{N}_{\mathbf{x}}=\mathbf{1 0 0}$ & $\mathbf{N}_{\mathbf{x}}=\mathbf{2 0 0}$ & $\mathbf{N}_{\mathbf{x}}=\mathbf{4 0 0}$ \\
\hline \multirow{3}{*}{$\mathbf{N}_{\boldsymbol{\delta}}=\mathbf{8}$} & $\mathbf{N}_{\gamma}=\mathbf{1}$ & 0.67840 & 0.67316 & 0.67050 & 0.49908 & 0.49733 & 0.49645 \\
& $\mathbf{N}_{\gamma}=\mathbf{4}$ & 0.63746 & 0.63223 & 0.62961 & 0.45458 & 0.45328 & 0.45262 \\
& $\mathbf{N}_{\gamma}=\mathbf{8}$ & 0.64305 & 0.63775 & 0.63508 & 0.45930 & 0.45792 & 0.45722 \\
& $\mathbf{N}_{\gamma}=\mathbf{1 6}$ & 0.64412 & 0.63879 & 0.63612 & 0.46016 & 0.45876 & 0.45805 \\
\hline \multirow{3}{*}{$\mathbf{N}_{\boldsymbol{\delta}}=\mathbf{1 6}$} & $\mathbf{N}_{\gamma}=\mathbf{1}$ & 0.71062 & 0.70430 & 0.70117 & 0.51616 & 0.51431 & 0.51338 \\
& $\mathbf{N}_{\gamma}=\mathbf{4}$ & 0.66572 & 0.65955 & 0.65649 & 0.46891 & 0.46763 & 0.46699 \\
& $\mathbf{N}_{\gamma}=\mathbf{8}$ & 0.67204 & 0.66575 & 0.66265 & 0.47415 & 0.47276 & 0.47208 \\
& $\mathbf{N}_{\gamma}=\mathbf{1 6}$ & 0.67333 & 0.66701 & 0.66389 & 0.47517 & 0.47376 & 0.47306 \\
\hline \multirow{3}{*}{$\mathbf{N}_{\boldsymbol{\delta}}=\mathbf{3 2}$} & $\mathbf{N}_{\gamma}=\mathbf{1}$ & 0.72273 & 0.71421 & 0.71020 & 0.52166 & 0.51925 & 0.51808 \\
& $\mathbf{N}_{\gamma}=\mathbf{4}$ & 0.67688 & 0.66848 & 0.66451 & 0.47363 & 0.47186 & 0.47101 \\
& $\mathbf{N}_{\gamma}=\mathbf{8}$ & 0.68346 & 0.67492 & 0.67088 & 0.47906 & 0.47717 & 0.47626 \\
& $\mathbf{N}_{\gamma}=\mathbf{1 6}$ & 0.68481 & 0.67625 & 0.67220 & 0.48013 & 0.47822 & 0.47730 \\
\hline
\end{tabular}

For the purpose of validation, since analytical solution is not available for a 2-D problem, LBM results must be compared with a numerical solution that could be considered as the reference one. In this work, LBM has been compared with the results obtained by the standard FVM. Validation 
analysis has been performed focusing on the dimensionless heat flux $\psi_{y}$. The error has been computed as follows:

$$
E r r=\frac{\left\|\psi_{y_{\text {FVMRef }}}-\psi_{y_{1 \mathrm{IM}}}\right\|_{2}}{\left\|\cdot \psi_{y_{\text {FWMRef }}}\right\|_{2}}
$$

where $\|\cdots\|_{2}$ indicates the Euclidean norm. In this work, the reference solution $\psi_{y_{\text {FVM,Ref }}}$ has been computed by means of a Richardson extrapolation of FVM results. In order to compute the reference solution, $N_{x}=160$ and $N_{\delta}=32$, in case of $\beta=2.0$, and $N_{x}=400$ and $N_{\delta}=32$, in case of $\beta=5.0$, have been assumed to be the reference spatial and azimuthal angle discretizations. Richardson extrapolation of the dimensionless heat flux has been performed for $N_{\gamma}=16$ and $N_{y}=32$. In the application of Eq. (27), the reference solution has been interpolated in order to match the grid points of the LBM solution. A cubic interpolation is used for not affecting the measurement of the order of the present numerical method.

For evaluating the order of convergence, let us recall Eq. (18b). Contributions to the global error of the LB scheme are given by the numerical approximation of the Lagrangian derivative of Eq. (9) and by the numerical approximation of the integrals over the solid angle. Eq. (18b) suggests that the model is first order accurate with respect to either the spatial or the azimuthal angle or the polar angle discretizations, for lattices which are small enough to solve accurately the advection step. However in case of large lattices, the streaming step of the lattice velocities belonging to larger energy shells, inevitably leads to an accuracy spoil. This trend can be demonstrated if $\Delta x, \Delta \delta$ and $\Delta \gamma$ are scaled by the same ratio during the grid refinement. In this paper the order of convergence of the method is evaluated with regard to the values of discretization parameters given in Table 2 . Errors of the dimensionless heat flux are shown in Tables 5, while the order of convergence can be evaluated from Figs. 7 and Table 6. It can be seen that the order of convergence of LB model decreases moving from case $\mathrm{B}$ to case $\mathrm{C}$ : this is ascribed to the accuracy spoil in the computation of the advection step for large lattices (case C uses the D2Q32 lattice), as already pointed out in the previous section.

The situation is even more critical for the polar discretization. In fact, from Table 5 one can notice that the error decreases as the spatial and azimuthal discretizations get more refined. The same trend does not show up for the polar discretization. Let us consider Figs 9a and 9b, where error is plotted as function of the infinitesimal polar angle $\Delta g=\pi / N_{\gamma}$ for $\beta=2.0$ and $\beta=5.0$. Subdivisions greater than $N_{\gamma}=1$ produce more accurate solutions, even though the minimum error occurs for 
$N_{\gamma}=4$. The larger the subdivisions in the polar direction, the closer the discrete polar angle $\gamma_{j}$ gets to the upper and lower bounds of the polar angle $\gamma$ : this makes intensity to move far outside from the 2-D lattice and it is like a fictitious increase in the free mean path of the radiation intensity. With other words, when the number of subdivisions of the polar angle increases, the magnitude $E_{i j}$ of the lattice velocities for $\gamma_{j}$ closest to 0 and $\pi$ become very large and this produces accuracy spoil. For these directions, the relaxation time (fictitiously) increases and then the distribution function gets closer to the equilibrium distribution function, as shown in Figs 8. This suggests another possible improvement. We enforce the intensities along $j$ directions closer to the z-axis to be equal to the equilibrium values and we call this technique thermalization. In Figs. 9a and $9 \mathrm{~b}$ the effects of this thermalization process are shown for $N_{\gamma}=8$ and $N_{\gamma}=16$. Thermalization produces an improvement in the accuracy of the solution with respect to the case where thermalization is not applied (Table 7), even if the best accuracy still occurs for $N_{\gamma}=4$. Results of Figs. $9 \mathrm{a}$ and $9 \mathrm{~b}$ have been obtained by thermalizing intensities at $\gamma_{2}=[\pi / 16,15 \pi / 16]$ in case of 8 subdivisions and $\left.\gamma_{j}=[\pi / 32,3 \pi / 32,5 \pi / 32,27 \pi / 32,29 \pi / 32,31 \pi / 32]\right]$ in case of 16 subdivisions.

Table 5. Errors of the dimensionless heat flux as function of the discretization and the extinction coefficients.

\begin{tabular}{|c|c|c|c|c|c|c|c|}
\cline { 3 - 8 } \multicolumn{2}{c|}{} & \multicolumn{3}{c|}{$\boldsymbol{\beta}=\mathbf{2 . 0}$} & \multicolumn{3}{c|}{$\boldsymbol{\beta}=\mathbf{5 . 0}$} \\
\cline { 3 - 8 } \multicolumn{2}{c|}{} & $\mathbf{N}_{\mathbf{x}}=\mathbf{4 0}$ & $\mathbf{N}_{\mathbf{x}}=\mathbf{8 0}$ & $\mathbf{N}_{\mathbf{x}}=\mathbf{1 6 0}$ & $\mathbf{N}_{\mathbf{x}}=\mathbf{1 0 0}$ & $\mathbf{N}_{\mathbf{x}}=\mathbf{2 0 0}$ & $\mathbf{N}_{\mathbf{x}}=\mathbf{4 0 0}$ \\
\hline \multirow{3}{*}{$\mathbf{N}_{\delta}=\mathbf{8}$} & $\mathbf{N}_{\gamma}=\mathbf{1}$ & 0.14875 & 0.13922 & 0.13618 & 0.13572 & 0.12638 & 0.12264 \\
& $\mathbf{N}_{\gamma}=\mathbf{4}$ & 0.10668 & 0.10216 & 0.10257 & 0.07615 & 0.07613 & 0.07811 \\
& $\mathbf{N}_{\gamma}=\mathbf{8}$ & 0.10693 & 0.10073 & 0.10029 & 0.07231 & 0.06991 & 0.07087 \\
& $\mathbf{N}_{\gamma}=\mathbf{1 6}$ & 0.10728 & 0.10069 & 0.10004 & 0.07200 & 0.06901 & 0.06970 \\
\hline \multirow{3}{*}{$\mathbf{N}_{\delta}=\mathbf{1 6}$} & $\mathbf{N}_{\gamma}=\mathbf{1}$ & 0.13591 & 0.11843 & 0.11129 & 0.14796 & 0.13389 & 0.12757 \\
& $\mathbf{N}_{\gamma}=\mathbf{4}$ & 0.06896 & 0.05687 & 0.05573 & 0.04789 & 0.04138 & 0.04219 \\
& $\mathbf{N}_{\gamma}=\mathbf{8}$ & 0.07306 & 0.05756 & 0.05412 & 0.05077 & 0.03922 & 0.03708 \\
& $\mathbf{N}_{\gamma}=\mathbf{1 6}$ & 0.07452 & 0.05837 & 0.05441 & 0.05236 & 0.03990 & 0.03706 \\
\hline \multirow{3}{*}{$\mathbf{N}_{\delta}=\mathbf{3 2}$} & $\mathbf{N}_{\gamma}=\mathbf{1}$ & 0.14174 & 0.11801 & 0.10759 & 0.15775 & 0.13937 & 0.13092 \\
& $\mathbf{N}_{\gamma}=\mathbf{4}$ & 0.06485 & 0.04356 & 0.03943 & 0.04546 & 0.03141 & 0.03032 \\
& $\mathbf{N}_{\gamma}=\mathbf{8}$ & 0.07175 & 0.04677 & 0.03887 & 0.05253 & 0.03301 & 0.02696 \\
& $\mathbf{N}_{\gamma}=\mathbf{1 6}$ & 0.07388 & 0.04832 & 0.03972 & 0.05502 & 0.03483 & 0.02791 \\
\hline
\end{tabular}

Table 6. Error and order of convergence of the LB scheme as function of the spatial discretization 


\begin{tabular}{|l|c|c|c|c|c|}
\hline \multicolumn{2}{|c|}{$\beta=\mathbf{2 . 0}$} & \multicolumn{3}{c|}{$\beta=5.0$} \\
\hline Spatial Discretization & Err & Order & Spatial Discretization & Err & Order \\
\hline A. $\mathrm{Nx}=40, \mathrm{~N}_{\delta}=8, \mathrm{~N}_{\gamma}=4$ & 0.10670 & - & A. $\mathrm{Nx}=100, \mathrm{~N}_{\delta}=8, \mathrm{~N}_{\gamma}=4$ & 0.07622 & - \\
& & & B. $\mathrm{Nx}=200, \mathrm{~N}_{\delta}=16, \mathrm{~N}_{\gamma}=$ & 0.03921 & 0.957 \\
B. $\mathrm{Nx}=80, \mathrm{~N}_{\delta}=16, \mathrm{~N}_{\gamma}=8$ & 0.05754 & 0.890 & 8 & & \\
C. $\mathrm{Nx}=160, \mathrm{~N}_{\delta}=32, \mathrm{~N}_{\gamma}=$ & 0.04826 & 0.535 & C. $\mathrm{Nx}=400, \mathrm{~N}_{\delta}=32, \mathrm{~N}_{\gamma}=$ & 0.02785 & 0.491 \\
16 & & & & 16 & \\
\hline
\end{tabular}

Table 7. Effects of thermalization on the accuracy for $\beta=2.0$ and $\beta=5.0$.

\begin{tabular}{|c|c|c|c|c|}
\cline { 2 - 5 } \multicolumn{1}{c|}{} & \multicolumn{2}{c|}{$\beta=\mathbf{2 . 0}, \mathbf{N}_{\mathbf{x}}=\mathbf{8 0}, \mathbf{N}_{\boldsymbol{\delta}}=\mathbf{3 2}$} & \multicolumn{2}{c|}{$\boldsymbol{\beta}=\mathbf{5 . 0}, \mathbf{N}_{\mathbf{x}}=\mathbf{2 0 0}, \mathbf{N}_{\boldsymbol{\delta}}=\mathbf{3 2}$} \\
\cline { 2 - 5 } & $\mathbf{N}_{\gamma}=\mathbf{8}$ & $\mathbf{N}_{\gamma}=\mathbf{1 6}$ & $\mathbf{N}_{\gamma}=\mathbf{8}$ & $\mathbf{N}_{\gamma}=\mathbf{1 6}$ \\
\hline No Thermalization & 0.04671 & 0.04826 & 0.03294 & 0.03474 \\
\hline Thermalization & 0.04419 & 0.04450 & 0.03119 & 0.03154 \\
\hline
\end{tabular}

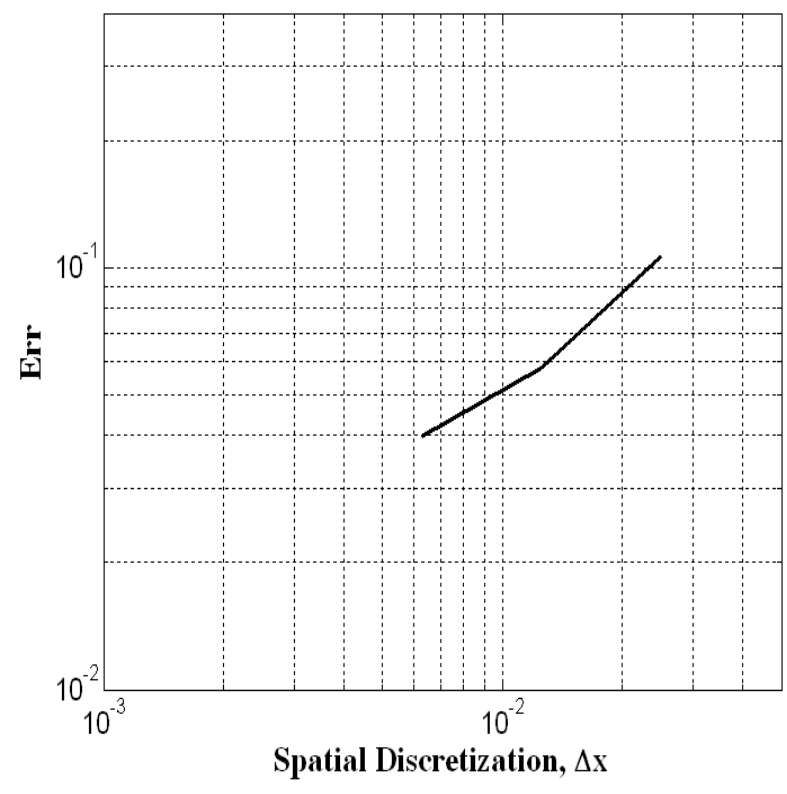

(a)

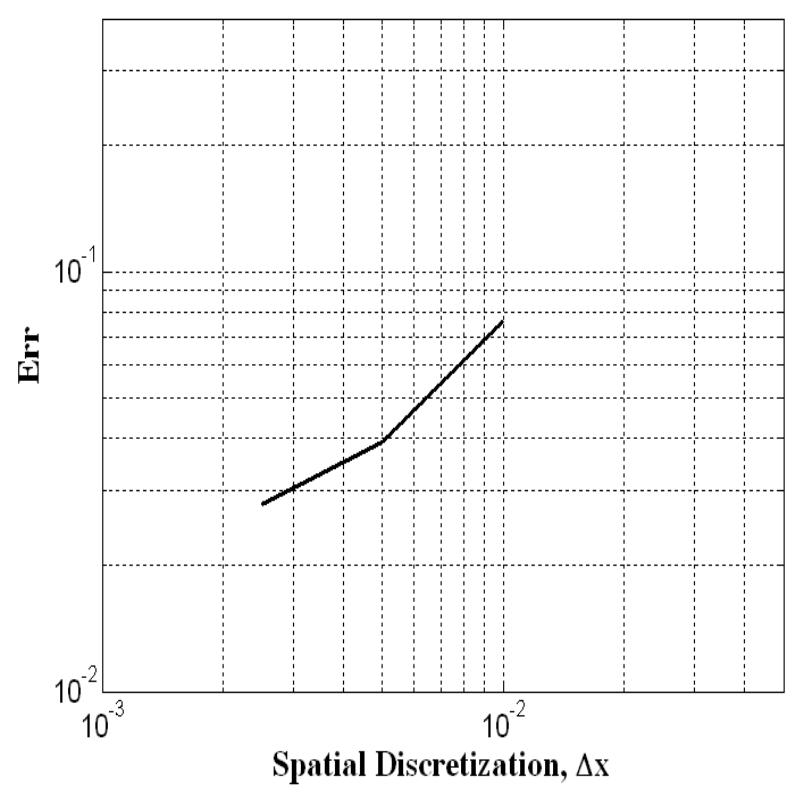

(b)

Figure 7. Order of convergence of the LB scheme as function of the spatial discretization for

$$
\boldsymbol{\beta}=\mathbf{2 . 0} \text { (a) and } \boldsymbol{\beta}=\mathbf{5 . 0}(\mathrm{b}) \text {. }
$$




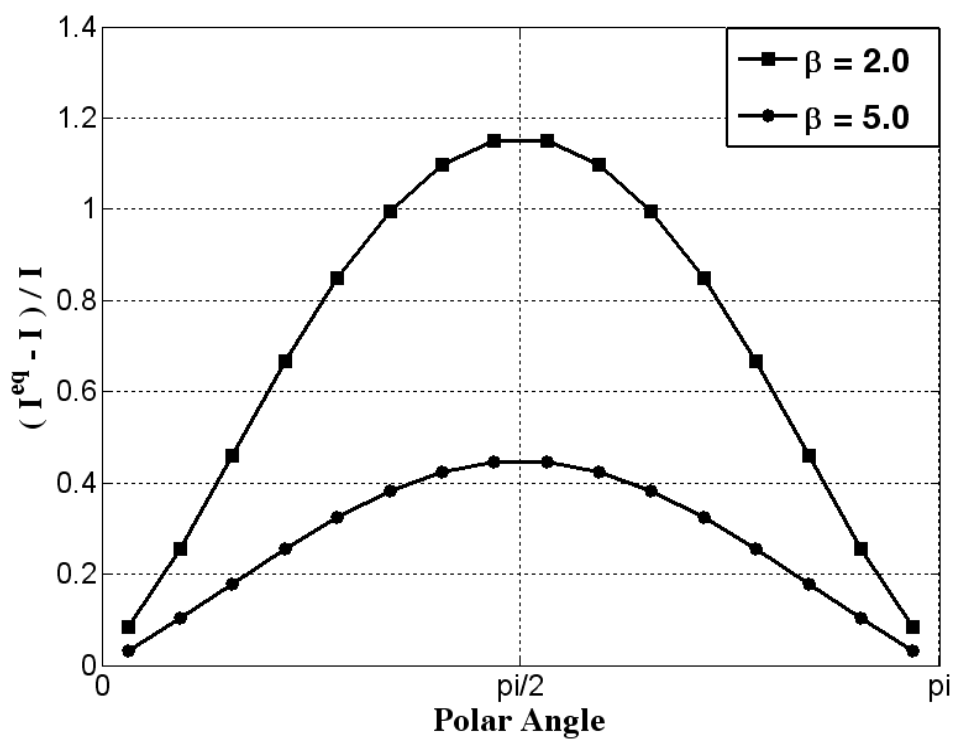

Figure 8. Comparison between PDF and the equilibrium PDF as function of the polar angle for $\beta=2.0$ and $\beta=5.0$.

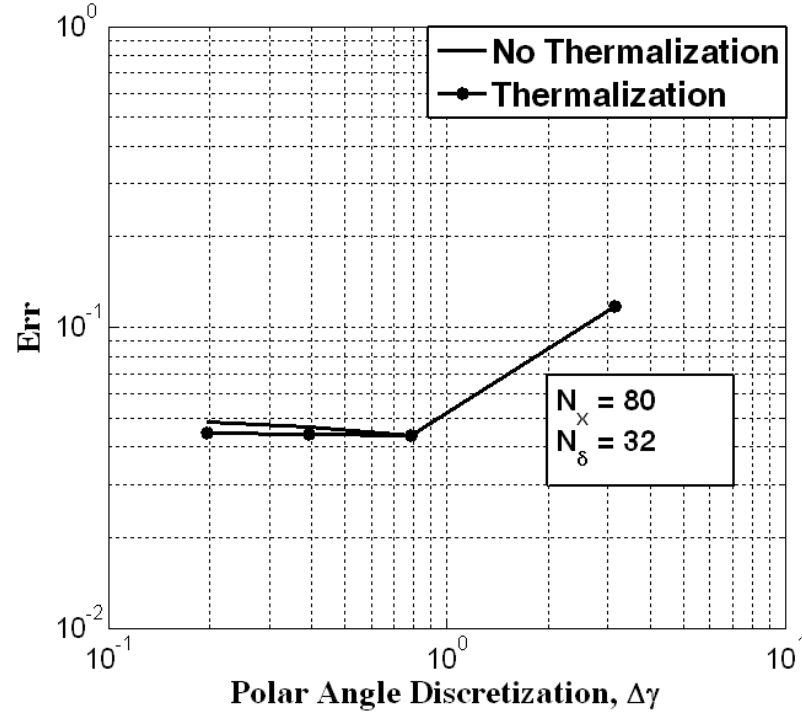

(a)

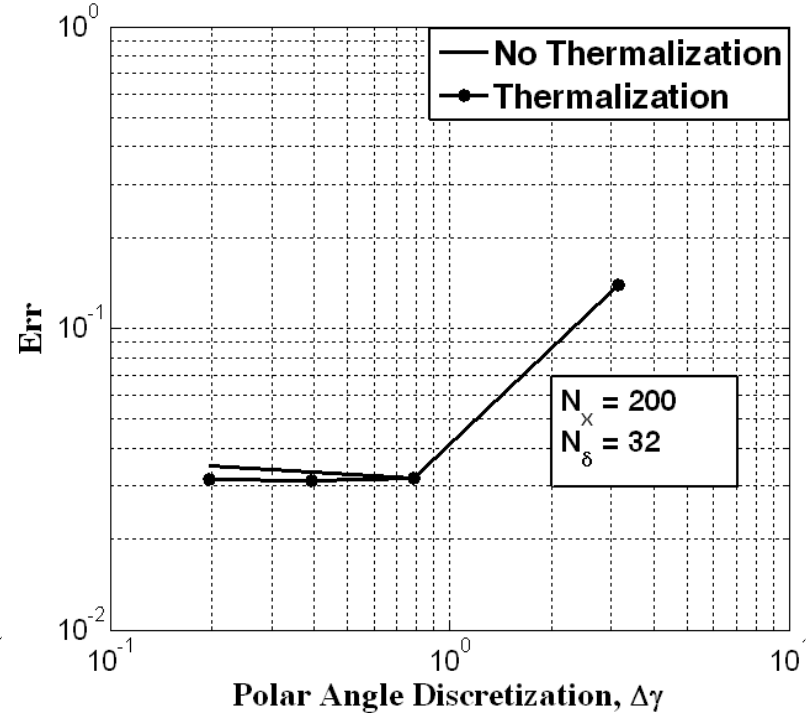

(b)

Figure 9. Error as function of the polar angle discretization for $\beta=2.0$ (a) and $\beta=5.0(b)$.

\section{CONCLUSIONS}

In this paper, some improvements to the lattice Boltzmann method (LBM) for solving radiative heat transfer in a participating medium, recently proposed by Asinari et al. [33], are presented and validated. In particular, the effects on the numerical solution produced by the discretization of the polar angle are systematically investigated. Essentially uniform discretization of the polar angle is possible by defining an enlarged set of lattice velocities, with an additional component along the 
axis normal to the main simulation plane. In this way, the projection of the total velocity still belongs to the original computational lattice.

A preliminary validation of the model has been considered by solving a benchmark radiative transfer problem in a 2-D rectangular enclosure. The results of the dimensionless heat flux obtained by the proposed LB scheme have been compared with a reference solution by standard FVM. Simulations have been performed for several values of the spatial and angular discretizations and extinction coefficients, in order to find out the order of convergence of the scheme. The numerical results demonstrate that the proposed radiative LB scheme is (at most) first order accurate, in case of compact lattices, which still ensure enough accuracy in the advection of the radiation information.

With regard to the effects of the discretization of the polar angle, we showed that minimum error occurs for a given subdivision, which does not necessarily correspond to the most refined discretization. This means that greater subdivisions produce a spoil in the numerical accuracy, due to larger (fictitious) mean free path of radiation. By means of a thermalization procedure, i.e. forcing the equilibrium values of some radiation intensities, a better accuracy can be achieved in case of large subdivisions of the polar angle.

\section{Acknowledgements}

SCM gratefully acknowledges the support of the Politecnico di Torino under the Visiting Professor Program of which he contributed to this work during his stay in Politecnico di Torino.

\section{APPENDIX}

The numerical results obtained by means of a standard FVM solver are reported for sake of completeness.

Table A.1. Mean temperature of the medium inside the enclosure computed through standard FVM.

\begin{tabular}{|c|c|c|c|c|c|c|c|}
\cline { 3 - 7 } \multicolumn{2}{c|}{} & \multicolumn{3}{c|}{$\boldsymbol{\beta = \mathbf { 2 . 0 }}$} & \multicolumn{3}{c|}{$\boldsymbol{\beta}=\mathbf{5 . 0}$} \\
\cline { 3 - 8 } \multicolumn{2}{c|}{} & $\mathbf{N}_{\mathbf{x}}=\mathbf{4 0}$ & $\mathbf{N}_{\mathbf{x}}=\mathbf{8 0}$ & $\mathbf{N}_{\mathbf{x}}=\mathbf{1 6 0}$ & $\mathbf{N}_{\mathbf{x}}=\mathbf{1 0 0}$ & $\mathbf{N}_{\mathbf{x}}=\mathbf{2 0 0}$ & $\mathbf{N}_{\mathbf{x}}=\mathbf{4 0 0}$ \\
\hline \multirow{3}{*}{$\mathbf{N}_{\delta}=\mathbf{8}$} & $\mathbf{N}_{\gamma}=\mathbf{1}$ & 0.64448 & 0.65976 & 0.66749 & 0.64674 & 0.65261 & 0.65554 \\
& $\mathbf{N}_{\gamma}=\mathbf{4}$ & 0.64461 & 0.65989 & 0.66759 & 0.64764 & 0.65349 & 0.65641 \\
& $\mathbf{N}_{\gamma}=\mathbf{8}$ & 0.64477 & 0.66004 & 0.66775 & 0.64790 & 0.65375 & 0.65667 \\
& $\mathbf{N}_{\gamma}=\mathbf{1 6}$ & 0.64483 & 0.66009 & 0.66780 & 0.64799 & 0.65383 & 0.65675 \\
\hline $\mathbf{N}_{\delta}=\mathbf{1 6}$ & $\mathbf{N}_{\gamma}=\mathbf{1}$ & 0.64627 & 0.66163 & 0.66938 & 0.64802 & 0.65390 & 0.65684 \\
& $\mathbf{N}_{\gamma}=\mathbf{4}$ & 0.64638 & 0.66173 & 0.66947 & 0.64893 & 0.65480 & 0.65773 \\
& $\mathbf{N}_{\gamma}=\mathbf{8}$ & 0.64655 & 0.66190 & 0.66964 & 0.64920 & 0.65507 & 0.65800
\end{tabular}




\begin{tabular}{|l|c|c|c|c|c|c|c|}
\hline & $\mathbf{N}_{\gamma}=\mathbf{1 6}$ & 0.64661 & 0.66195 & 0.66969 & 0.64929 & 0.65515 & 0.65808 \\
\hline \multirow{3}{*}{$\mathbf{N}_{\boldsymbol{\delta}} \mathbf{3 2}$} & $\mathbf{N}_{\gamma}=\mathbf{1}$ & 0.64665 & 0.66207 & 0.66984 & 0.64830 & 0.65420 & 0.65714 \\
& $\mathbf{N}_{\gamma}=\mathbf{4}$ & 0.64677 & 0.66218 & 0.66993 & 0.64922 & 0.65510 & 0.65804 \\
& $\mathbf{N}_{\gamma}=\mathbf{8}$ & 0.64693 & 0.66234 & 0.67010 & 0.64949 & 0.65537 & 0.65830 \\
& $\mathbf{N}_{\gamma}=\mathbf{1 6}$ & 0.64699 & 0.66239 & 0.67015 & 0.64957 & 0.65545 & 0.65839 \\
\hline
\end{tabular}

Table A.2. Dimensionless total heat flux along the south wall computed through standard FVM.

\begin{tabular}{|c|c|c|c|c|c|c|c|}
\cline { 3 - 8 } \multicolumn{2}{c|}{} & \multicolumn{3}{c|}{$\boldsymbol{\beta = \mathbf { 2 . 0 }}$} & \multicolumn{3}{c|}{$\boldsymbol{\beta}=\mathbf{5 . 0}$} \\
\cline { 3 - 8 } \multicolumn{1}{c|}{} & $\mathbf{N}_{\mathbf{x}}=\mathbf{4 0}$ & $\mathbf{N}_{\mathbf{x}}=\mathbf{8 0}$ & $\mathbf{N}_{\mathbf{x}}=\mathbf{1 6 0}$ & $\mathbf{N}_{\mathbf{x}}=\mathbf{1 0 0}$ & $\mathbf{N}_{\mathbf{x}}=\mathbf{2 0 0}$ & $\mathbf{N}_{\mathbf{x}}=\mathbf{4 0 0}$ \\
\hline \multirow{3}{*}{$\mathbf{N}_{\boldsymbol{\delta}}=\mathbf{8}$} & $\mathbf{N}_{\gamma}=\mathbf{1}$ & 0.65157 & 0.65246 & 0.65288 & 0.45370 & 0.45413 & 0.45434 \\
& $\mathbf{N}_{\gamma}=\mathbf{4}$ & 0.65768 & 0.65855 & 0.65899 & 0.46249 & 0.46293 & 0.46315 \\
& $\mathbf{N}_{\gamma}=\mathbf{8}$ & 0.66000 & 0.66088 & 0.66131 & 0.46520 & 0.46565 & 0.46587 \\
& $\mathbf{N}_{\gamma}=\mathbf{1 6}$ & 0.66075 & 0.66163 & 0.66206 & 0.46605 & 0.46650 & 0.46672 \\
\hline \multirow{3}{*}{$\mathbf{N}_{\boldsymbol{\delta}}=\mathbf{1 6}$} & $\mathbf{N}_{\gamma}=\mathbf{1}$ & 0.66101 & 0.66191 & 0.66236 & 0.46355 & 0.46400 & 0.46421 \\
& $\mathbf{N}_{\gamma}=\mathbf{4}$ & 0.66695 & 0.66783 & 0.66829 & 0.47236 & 0.47281 & 0.47303 \\
& $\mathbf{N}_{\gamma}=\mathbf{8}$ & 0.66923 & 0.67012 & 0.67057 & 0.47508 & 0.47553 & 0.47576 \\
& $\mathbf{N}_{\gamma}=\mathbf{1 6}$ & 0.66996 & 0.67085 & 0.67131 & 0.47594 & 0.47639 & 0.47662 \\
\hline \multirow{3}{*}{$\mathbf{N}_{\boldsymbol{\delta}}=\mathbf{3 2}$} & $\mathbf{N}_{\gamma}=\mathbf{1}$ & 0.66352 & 0.66437 & 0.66483 & 0.46613 & 0.46654 & 0.46676 \\
& $\mathbf{N}_{\gamma}=\mathbf{4}$ & 0.66940 & 0.67027 & 0.67072 & 0.47495 & 0.47537 & 0.47560 \\
& $\mathbf{N}_{\gamma}=\mathbf{8}$ & 0.67168 & 0.67254 & 0.67300 & 0.47767 & 0.47810 & 0.47833 \\
& $\mathbf{N}_{\gamma}=\mathbf{1 6}$ & 0.67241 & 0.67328 & 0.67373 & 0.47853 & 0.47896 & 0.47919 \\
\hline
\end{tabular}

\section{REFERENCES}

[1]. J. G. Marakis, C. Papapavlou, and Kakaras, E., A Parametric Study of Radiative Heat Transfer in Pulverised Coal Furnaces, Int. J. Heat Mass Transfer, vol. 43, pp. 2961-2971, 2000.

[2]. M. Spinnler, E. R. F. Winter, and R. Viskanta, Studies on High-Temperature Multilayer Thermal Insulations, Int. J. Heat Mass Transfer, vol. 47, pp. 1305-1312, 2004.

[3]. C. Yao, G.X. Wang, and B.T.F. Chung, Nonequilibrium Planar Interface Model for Solidification of Semitransparent Radiating Materials. J. Thermophys Heat Transfer, vol. 14, pp. 297-304, 2000.

[4]. Y. Shu, B.Q. Al, L. A1, and K.G. Lynn, Numerical Modeling of Internal Radiation and Solidification in Semitransparent Melts in Magnetic Fields, Numer. Heat Transfer B, vol. 45, pp. 957-976, 2004. 
[5]. P. Sadooghi, Transient Coupled Radiative and Conductive Heat Transfer in a Semitransparent Layer of Ceramic, J. Quant. Spectros. Radiat. Transfer, vol. 92, pp. 403416, 2005.

[6]. R. Raj, A. Prasad, P.R. Parida, and S.C. Mishra, Analysis of Solidification of a Semitransparent Planar Layer using the Lattice Boltzmann Method and the Discrete Transfer Method, Numer. Heat Transfer A, vol. 49, pp. 279-299, 2006,

[7]. W. W. Grabowski, Impact of Cloud Microphysics on Convective-Radiative Quasi Equilibrium Revealed by Cloud-resolving Convection Parameterization, J. Clim. vol. 16, pp. 3463-3475, 2003.

[8]. K. Kim and Z. Guo, Ultrafast Radiation Heat Transfer in Laser Tissue Welding and Soldering, Numer. Heat Transfer A, vol. 46, pp.23-40, 2004.

[9]. G. Pal, S. Basu, K. Mitra, and S. Kumar, Bioheat Transfer in Layered Skin Model Subjected to Short Pulse Laser Irradiation, Laser Surg. Med., vol. 13, pp. 12-37, 2005.

[10]. R. Siegel and J. R. Howell, Thermal Radiation Heat Transfer, fourth ed., Taylor \& Francis, New York, 2002.

[11]. M.F. Modest, Radiative Heat Transfer, second ed., Academic Press, New York, 2003.

[12]. W.A. Fiveland, Discrete-Ordinates Solution of the Radiative Transport Equation for Rectangular Enclosures, J. Heat Transfer, vol. 106, pp. 699-706, 1984.

[13]. S.C. Mishra, H.K. Roy, and N. Misra, Discrete Ordinate Method with a New and a Simple Quadrature Scheme, J. Quant. Spectrosc. Radiat. Transfer, vol. 101, pp. 249-262, 2006.

[14]. F.C. Lockwood, and N.G. Shah, A New Radiation Solution Method for Incorporation in General Combustion Prediction Procedures. In: Eighteenth Symposium (Int.) on Combustion, The Combustion Institute, Pittsburgh, pp. 1405-1414, 1981.

[15]. P.S. Cumber and M. Fairweather, Evaluation of Flame Emission Models Combined with the Discrete Transfer Method for Combustion System Simulation, Int. J. Heat Mass Transfer, vol. 48, pp. 5221-5239. 2005.

[16]. N. Anand and S.C. Mishra, The Discrete Transfer Method Applied to the Radiative Heat Transfer in a Variable Refractive Index Semitransparent Medium, J. Quant. Spectrosc. Radiat. Transfer, vol. 102, pp. 432-440, 2006.

[17]. S.C. Mishra and M. Prasad, Radiative Heat Transfer in Absorbing-Emitting-Scattering Gray Media Inside 1-D Gray Cartesian Enclosure using the Collapsed Dimension Method, Int. J. Heat Mass Transfer, vol. 45, pp. 697-700. 2002. 
[18]. J.C. Chai and S.V. Patankar, Finite Volume Method for Radiation Heat Transfer, Adv. Numerical Heat Transfer, vol. 2, pp. 110-135, 2000.

[19]. M.Y. Kim, S.W. Baek, and C.Y. Lee, Prediction of Radiative Heat Transfer Between Two Concentric Spherical Enclosures with the Finite Volume Method. Int. J. Heat Mass Transfer, vol. 51, pp. 4820-4828, 2008.

[20]. S.C. Mishra and H.K. Roy, Solving Transient Conduction-Radiation Problems using the Lattice Boltzmann Method and the Finite Volume Method, J. Comput. Physics, vol. 223, pp. 89-107, 2007.

[21]. S. Chen and G.D. Doolen, Lattice Boltzamann Method for Fluid Flows, Annu. Rev. Fluid Mech., vol. 30, pp. 329-364. 1998.

[22]. S. Succi, The Lattice Boltzmann Method for Fluid Dynamics and Beyond, Oxford University Press, New York, 2001.

[23]. F. Massaioli, R. Benzi, and S. Succi, Exponential Tails in Rayleigh-Benard Convection, Europhys. Lett., vol. 21, pp. 305-310, 1993.

[24]. J.G.M. Eggels and J.A. Sommers, Numerical Simulation of Free Convective Flow using the Lattice-Boltzmann Scheme, Int. J. Heat Fluid Flow, vol. 16, pp. 357-364, 1995.

[25]. X. Shan, Simulation of Rayleigh-Bénard Convection using a Lattice Boltzmann Method, Phys. Rev. E, vol. 55, pp. 2780-2788, 1997.

[26]. S. Succi, G. Bella, and F. Papetti, Lattice Kinetic Theory for Numerical Combustion, J. Sci. Comput. vol. 12, pp. 395-408, 1997.

[27]. X. He, S. Chen, and G.D. Doolen, A Novel Thermal Model for the Lattice Boltzmann Method in Incompressible Limit, J. of Comp. Physics, vol. 146, pp. 282-300, 1998.

[28]. R.G.M. van der Sman, M.H. Ernst, and A.C. Berkenbosch, Lattice Boltzmann Scheme for Cooling of Packed Cut Flowers, Int. J. Heat Mass Transfer, vol. 43, pp. 577-587, 2000.

[29]. S.C. Mishra, A. Lankadasu, and K. Beronov, Application of the Lattice Boltzmann Method for Solving the Energy Equation of a 2-D Transient Conduction-Radiation Problem, Int. J. Heat Mass Transfer, vol. 48, pp. 3648-3659. 2005.

[30]. R. Das, S.C. Mishra, M. Ajith, and R. Uppaluri, An Inverse Analysis of a Transient 2-D Conduction-Radiation Problem Using the Lattice Boltzmann Method and the Finite Volume Method Coupled with the Genetic algorithm. J. Quant. Spectrosc. Radiat. Transfer, vol. 109, pp. 2060-2077, 2008. 
[31]. S.C. Mishra, T.B. Pavan Kumar, and B. Mondal, Lattice Boltzmann Method Applied to the Solution of Energy Equation of a Radiation and Non-Fourier Heat Conduction Problem, Numer. Heat Transfer A, vol. 54, pp. 798-818, 2008.

[32]. B. Mondal, and S.C. Mishra, Simulation of Natural Convection in the Presence of Volumetric Radiation Using the Lattice Boltzmann Method, Numer. Heat Transfer A, vol. 55, pp. 18-41, 2009.

[33]. P. Asinari, S. C. Mishra, R. Borchiellini, A Lattice Boltzmann formulation to the analysis of radiative heat transfer problems in a participating medium, Numer. Heat Transfer A, in press, 2010. 